\title{
SAÚDE AUDITIVA DOS RECÉM-NASCIDOS: ATUAÇÃO DA FONOAUDIOLOGIA NA ESTRATÉGIA SAÚDE DA FAMÍLIA
}

\author{
Newborn hearing health: speech therapy \\ acting on family health strategy
}

\author{
Raquel Martins Maia( ${ }^{(1)}$, Maria Adelane Monteiro da Silva(2), Patrícia Moreira Bezerra Tavares ${ }^{(3)}$
}

\begin{abstract}
RESUMO
Objetivo: analisar o acompanhamento dos recém-nascidos quanto à promoção da saúde auditiva após a inserção da fonoaudiologia na Estratégia Saúde da Família. Método: estudo retrospectivo e documental com abordagem quantitativa com 88 recém-nascidos que realizaram o teste da orelhinha, no período de fevereiro a maio de 2010, a partir dos relatórios mensais de devolutiva do Serviço de Atenção a Saúde Auditiva do município, consolidados mensais e prontuários de um Centro de Saúde da Família em Sobral-Ce. Resultados: dos recém-nascidos avaliados, 35 (39,77\%) falharam no teste, entre estes, $7(20 \%)$ apresentam indicador de risco para deficiência auditiva e $28(80 \%)$ não apresentavam nenhum risco. Verificou-se também divergências entre os dados do Serviço de Atenção a Saúde Auditiva e os prontuários do Centro de Saúde da Família quanto a classificação dos indicadores de risco para a perda auditiva. Observou-se ainda que, o número de encaminhamentos para o teste da orelhinha aumentou $8,33 \%$. Em relação ao reteste, $1(7,69 \%)$ criança retornou nos meses de março a agosto de 2009 e entre os meses de setembro/2009 a fevereiro/2010 após a atuação da fonoaudiologia no CSF do Sumaré 17 (65,38\%) crianças realizaram o reteste. Conclusão: os dados sugerem a importância da presença do fonoaudiólogo na atenção primária, sendo fundamental no acompanhamento e monitoramento do diagnóstico precoce das alterações auditivas.
\end{abstract}

DESCRITORES: Promoção da Saúde; Audição; Fonoaudiologia

\section{INTRODUÇÃO}

A Prefeitura Municipal de Sobral, para garantir a assistência às pessoas com deficiência auditiva, implementou em 2002 o Projeto Escuta Sobral ${ }^{1}$ e o Serviço de Atenção à Saúde Auditiva (SASA), que

(1) Fonoaudióloga; Graduada pela Faculdade de Saúde, Ciências Humanas e Tecnológicas do Piauí, Teresina-Pi; Especialização com Caráter de Residência Multiprofissional em Saúde da Família pela Escola de Formação em Saúde da Família Visconde de Sabóia, Sobral-CE e Universidade Estadual Vale do Acaraú, Sobral-CE.

(2) Enfermeira; Docente da Escola de Formação em Saúde da Família Visconde de Sabóia, Sobral-CE; Doutora em Enfermagem pela Universidade Federal do Ceará, Fortaleza-CE.

(3) Fonoaudióloga; Serviço de Atenção à Saúde Auditiva, Sobral-CE; Especialização em Audiologia pelo Núcleo de Tratamento e Estimulação Precoce pela Universidade Federal do Ceará, Fortaleza-CE; Especialização em Motricidade Orofacial pelo CEFAC-Saúde e Educação.

Conflito de interesses: inexistente em 2005 passou a ser referência para a atenção primária e secundária à saúde do município e macrorregião. Atualmente o projeto realiza várias atividades, entre elas destaca-se a importância da Triagem Auditiva Neonatal (TAN), tendo em vista a possibilidade de detecção e intervenção precoce da deficiência auditiva.

A audição desempenha um papel preponderante e decisivo na aquisição e desenvolvimento de fala e linguagem. Desta forma, a detecção precoce das alterações auditivas é essencial para prevenir ou minimizar os riscos e desvios que possam interferir no desenvolvimento da criança.

O início da audição ocorre por volta da 20a semana de gestação e se desenvolve ativamente nos primeiros meses de vida. Existe uma ordem de sucessão no desenvolvimento da fala - o início do arrulho, risadas, e o balbucio repetido segue o início da vocalização e acaba levando à produção de palavras simples ${ }^{2}$. Qualquer interrupção em 
algumas dessas etapas, especialmente as iniciais, acarretará prejuízos importantes no desenvolvimento de fala e linguagem da criança.

Estima-se que no Brasil 3 a 5 crianças em 1.000 nascem surdas, aumentando para 2 a 4 em cada 100 nascimentos quando provenientes de Unidade de Terapia Intensiva (UTI). A idade média do diagnóstico da deficiência auditiva varia em torno de 3 a 4 anos de idade, podendo levar até 2 anos para ser concluído ${ }^{3}$. Portanto, a intervenção precoce deve ser iniciada antes de seu primeiro ano de vida, pois resulta em um melhor desenvolvimento da fala e linguagem, a aquisição de habilidades cognitivas, o desenvolvimento sócio-emocional e ao sucesso acadêmico ${ }^{2}$.

A TAN tem o objetivo de detectar precocemente alterações auditivas em recém-nascidos (RN) por meio das Emissões Otoacústicas (EOA), ou seja, o teste da orelhinha. As EOA são energias sonoras de fraca intensidade que são amplificadas pela contração das células ciliadas externas, na cóclea, podendo ser captadas no Conduto Auditivo Externo (CAE) ${ }^{4}$. O método das EOA é simples, de rápida realização, pode ser aplicado durante o sono fisiológico e não requer sedação.

As intercorrências pré, peri e pós-natais caracterizam os Indicadores de Risco para Deficiência Auditiva (IRDA). Os IRDA são: permanência em UTI por mais de 48hs; asfixia perinatal grave; meningite bacteriana; infecções intra-uterinas (sífilis, toxoplasmose, rubéola, citomegalovírus e herpes); anomalias crâniofaciais, incluindo anormalidades morfológicas do pavilhão auricular e do CAE; hiperbilirrubinemia/exsangüíneo transfusão; história familiar de perda auditiva; peso inferior a 1.500g; ventilação mecânica por mais de 5 dias; síndromes associadas à perda auditiva condutiva ou neurossensorial; medicação ototóxica, incluindo aminoglicosídeos usados por mais de 5 dias e diuréticos usados em combinação com os aminoglicosídeos ${ }^{5}$.

A importância da pesquisa desses indicadores está relacionada principalmente ao fato de que a audição normal ao nascer não impede o início tardio de perda auditiva e/ou a progressão da perda auditiva já existente ao nascimento, bem como a observação e acompanhamento de crianças que não apresentaram ao nascer nenhum dos IRDA, mas podem desenvolver uma deficiência auditiva.

Pensando nisso, o SASA em articulação com a Atenção Primária à Saúde (APS) passou a desenvolver programas de promoção à saúde auditiva. É na APS que se inserem os profissionais da Residência Multiprofissional em Saúde da Família - RMSF (Fisioterapia, Terapia Ocupacional,
Psicologia, Serviço Social, Educação Física, Nutrição, Farmácia, Fonoaudiologia, Enfermagem e Odontologia), que tem a Escola de Formação em Saúde da Família Visconde de Sabóia como responsável pela execução pedagógica, bem como os profissionais da equipe de saúde da atenção básica, em especial médicos e enfermeiras, que são habilitados para identificar os IRDA da gestação até o puerpério.

Atualmente o município possui 5 fonoaudiólogas residentes distribuídas pelos Centros de Saúde da Família (CSF) na sede do município. A fonoaudiologia inserida na Estratégia Saúde da Família (ESF) passou a atuar no acompanhamento e monitoramento dos RN a partir de setembro de 2009 por meio de visitas domiciliares às crianças que haviam falhado no teste da orelhinha para reforçar a importância da TAN, o prazo dos retestes e os cuidados com a audição. Essas crianças eram revisitadas, caso falhassem novamente.

A relevância deste estudo se justifica por oportunizar a discussão entre fonoaudiólogos e equipe mínima sobre a necessidade do diagnóstico precoce das alterações auditivas em RN até os seis meses de vida, bem como o fortalecimento/concretização do SASA no município, consolidando o papel do fonoaudiólogo na APS como sendo fundamental para garantir o acompanhamento das crianças no diagnóstico precoce da deficiência auditiva, propiciando melhor qualidade de vida da população infantil no município de Sobral.

Portanto, esta pesquisa teve como objetivos: analisar o acompanhamento dos $\mathrm{RN}$ quanto à promoção da saúde auditiva após a inserção da fonoaudiologia na ESF; verificar a incidência dos $\mathrm{RN}$ que falharam no teste da orelhinha no período de janeiro a dezembro de 2009; caracterizar os RN que falharam no teste da orelhinha nesse mesmo período e comparar a média de retornos dos $\mathrm{RN}$ antes e após a inserção da fonoaudiologia na ESF, no período março/2009 a fevereiro/2010.

\section{MÉTODO}

Trata-se de um estudo retrospectivo e documental com abordagem quantitativa realizado no período de fevereiro a maio de 2010, no CSF do bairro Sumaré no município de Sobral-CE.

O Município de Sobral situado na Região Noroeste do Estado do Ceará, a 230 km da capital Fortaleza, por via da BR-222, apresenta uma extensão territorial de $2.129 \mathrm{~km}^{2}$ com uma população de 182.431 habitantes ${ }^{6}$. Ali, estão localizados os bairros Sumaré e Padre Palhano. 
Constituiu-se cenário do estudo apenas o bairro Sumaré em razão da disponibilidade da equipe básica nas ações referentes à saúde auditiva, bem como a acessibilidade às informações contidas nos prontuários da família, o que possibilitou uma análise de dados mais fidedigna com a realidade local.

O CSF Cleide Cavalcante Sales no bairro Sumaré, possui 1.938 famílias atendidas por duas equipes de saúde, sendo que $4,54 \%$ dos habitantes estão na faixa etária menor de 1 ano e 22,29\% estão entre 1 a 4 anos ${ }^{7}$.

Constituíram sujeitos do estudo $88 \mathrm{RN}$ que realizaram o Teste da Orelhinha no ano de 2009 no SASA. O critério de inclusão na pesquisa foi a realização do teste no período do estudo.

Para coleta de dados referentes à incidência, caracterização e acompanhamento dos RN que realizaram o teste da orelhinha no período de janeiro a dezembro/2009 foram pesquisados os relatórios mensais de devolutiva do SASA ao CSF Sumaré e os consolidados mensais das unidades e para tanto, elaborou-se como instrumento uma planilha.

Também foram analisados os prontuários da família no referido CSF para caracterização desses $\mathrm{RN}$, coletados a partir de um formulário. O acesso aos prontuários foi previamente autorizado por meio do Termo de Autorização: Materiais Arquivados (Prontuários).

O projeto do estudo foi submetido ao Comitê de Ética em Pesquisa da Universidade Estadual do Vale do Acaraú - UVA e aprovado sob o no 834.

A análise estatística é do tipo descritiva e está apresentada nas figuras e tabelas. O estudo teve como aporte teórico para a análise a literatura pertinente ao tema sobre a TAN, prevalência da surdez no Brasil e os IRDA.

\section{RESULTADOS}

No ano de 2009 foram avaliados $88 \mathrm{RN}$, sendo que $35(39,77 \%)$ falharam no teste da orelhinha, percebendo que a incidência de falha apresenta-se

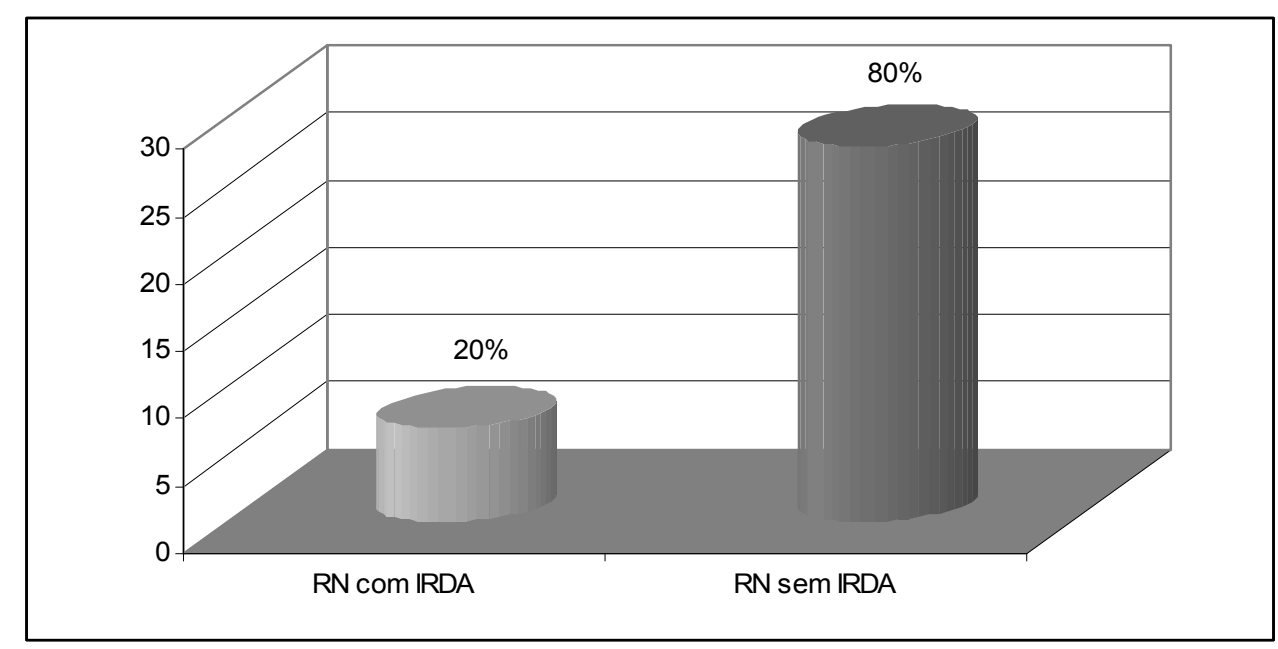

Figura 1 - Distribuição porcentual dos RN que falharam no teste da orelhinha no ano de 2009 no município de Sobral-CE, segundo ausência ou presença de indicador de risco. Sobral-CE, 2010

elevada. A figura 1 mostra que dos $35(39,77 \%)$ RN que falharam no teste da orelhinha, 7 (20\%) são RN com IRDA e 28 (80\%) não apresentam nenhum IRDA.

Em relação a caracterização dos $\mathrm{RN}$ que falharam no teste da orelhinha a tabela 1 aponta que de acordo com o sexo, $62,86 \%$ são do sexo masculino e $37,14 \%$ do sexo feminino. Em relação ao tipo de parto, $42,86 \%$ foi normal, $51,43 \%$ cesárea e $5,71 \%$ não continham registro do tipo de parto. Segundo a idade gestacional, $22,86 \%$ nasceram de
30 a 37 semanas, $51,43 \%$ de 38 a 41 semanas e $25,71 \%$ não haviam registro da idade gestacional dos bebês.

A tabela 2 classifica os RN com IRDA segundo o tipo de indicador de risco encontrados nos prontuários e relatórios pesquisados. São apresentados os indicadores de risco: ototoxidade $25,71 \%$, prematuridade $17,14 \%$, hiperbilirrubinemia $2,86 \%$, anóxia $2,86 \%$, peso $<1500 \mathrm{~g} 2,86 \%$, peso $<2500 \mathrm{~g} 5,71 \%$, sífilis congênita $2,86 \%$. 
Tabela 1 - Caracterização dos RN que falharam no teste da orelhinha de acordo com a idade gestacional, tipo de parto e sexo no ano de 2009 no município de Sobral-CE. Sobral-CE, 2010

\begin{tabular}{lccccc}
\hline \multirow{2}{*}{ Características } & \multicolumn{2}{c}{ Sem Risco Auditivo } & \multicolumn{2}{c}{ Com Risco Auditivo } & \multirow{2}{*}{ Total } \\
\cline { 2 - 5 } & $\mathbf{N}$ & $\%$ & $\mathbf{N}$ & $\%$ & \\
\cline { 1 - 4 } Sexo & 17 & $(77,28)$ & 5 & $(22,72)$ & 22 \\
Masculino & 11 & $(84,62)$ & 2 & $(15,38)$ & 13 \\
Feminino & & & & & \\
Tipo de Parto & 12 & $(80,00)$ & 3 & $(20,00)$ & 15 \\
Normal & 14 & $(77,78)$ & 4 & $(22,22)$ & 18 \\
Cesárea & 2 & $(100,00)$ & - & & 2 \\
Não informado & 5 & $(62,50)$ & 3 & $(37,50)$ & 8 \\
Idade Gestacional & 16 & $(88,90)$ & 2 & $(11,10)$ & 18 \\
30 a 37 semanas & 7 & $(77,80)$ & 2 & $(22,20)$ & 9 \\
38 a 41 semanas & & & & & \\
Não informado & & & & & \\
\hline
\end{tabular}

Tabela 2 - Classificação dos RN segundo o indicador de risco no ano de 2009, no município de Sobral-CE. Sobral-CE, 2010

\begin{tabular}{lcc}
\hline Indicador de Risco & $\mathbf{N}^{\circ} \mathbf{R N}$ classificado com IRDA & $\mathbf{N}^{\circ}$ RN classificado com IRDA \\
\hline Ototoxidade & 4 & 5 \\
Prematuridade & 3 & 3 \\
Hiperbilirrubinemia & 1 & - \\
Anóxia & - & 1 \\
Peso $<1500 \mathrm{~g}$ & 1 & - \\
Peso $<2500 \mathrm{~g}$ & - & 2 \\
Sífilis Congênita & - & 1 \\
\hline
\end{tabular}

*Classificação segundo relatório mensal de devolutiva do SASA.

**Classificação segundo os prontuários do RN no CSF.

Segundo o acompanhamento dos RN pela fonoaudiologia na ESF, a figura 2 mostra que no período de janeiro a agosto/2009, dos 46 RN encaminhados para o teste da orelhinha pelos profissionais da ESF, apenas 37 realizaram o teste. Em comparação aos meses de setembro a dezembro/2009, das 50 crianças encaminhadas, 65 realizaram o teste da orelhinha, implicando num aumento de 8,33\% após o início da atuação da fonoaudiologia em setembro/2009.

Em relação ao acompanhamento e monitoramento dos $\mathrm{RN}$, nos meses de março a agosto de 2009, das 13 crianças que falharam no teste apenas 1 (7,69\%) criança retornou. Já em relação aos meses de setembro/2009 a fevereiro/2010 após a atuação da residente de fonoaudiologia no CSF do Sumaré, das 26 crianças que falharam no teste $17(65,38 \%)$ voltaram para o reteste, conforme a figura 3.

\section{DISCUSSÃO}

Verificou-se que no ano de 2009 dos RN avaliados, $39,77 \%$ falharam no teste (esta porcentagem engloba $\mathrm{RN}$ com e sem risco auditivo). Para os critérios de "passa/falha" para o teste da orelhinha usa-se um escore de reprodutibilidade de $50 \%$, ou mais, nas freqüências de 1000 a $4000 \mathrm{~Hz}^{2}$. Se durante o exame, a criança não atingir o mínimo de $50 \%$ em três das cinco bandas de freqüências, significa que "falhou" no teste.

É importante esclarecer também que o "passa" no teste equivale dizer que, no momento do teste, os resultados foram compatíveis com audição normal. Isso não significa que no decorrer da infância, perdas auditivas não possam ser adquiridas por otite secretora, infecções, medicamentos ototóxicos, causas genéticas ou traumáticas, podendo gerar perdas auditivas permanentes ${ }^{8}$. 


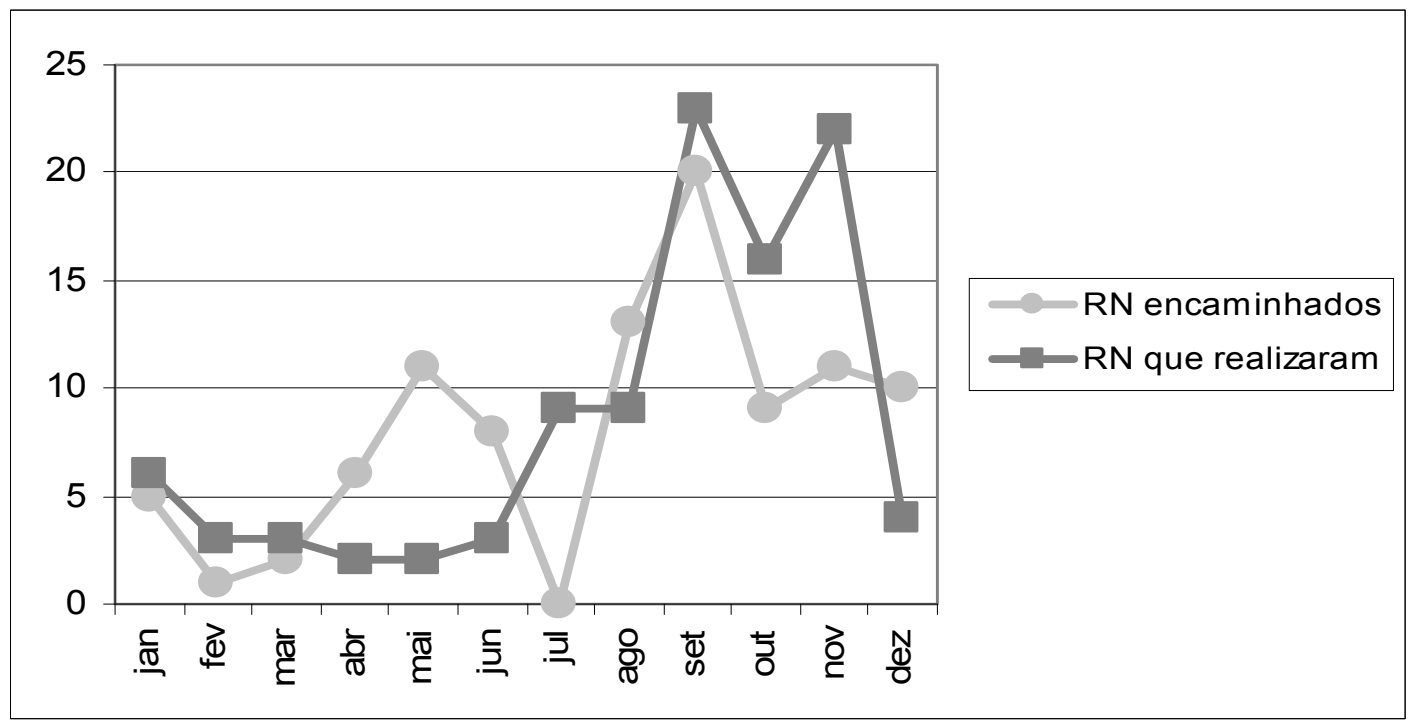

Figura 2 - Distribuição dos RN encaminhados e que realizaram o teste da orelhinha no ano de 2009 no município de Sobral-CE. Sobral-Ce, 2010

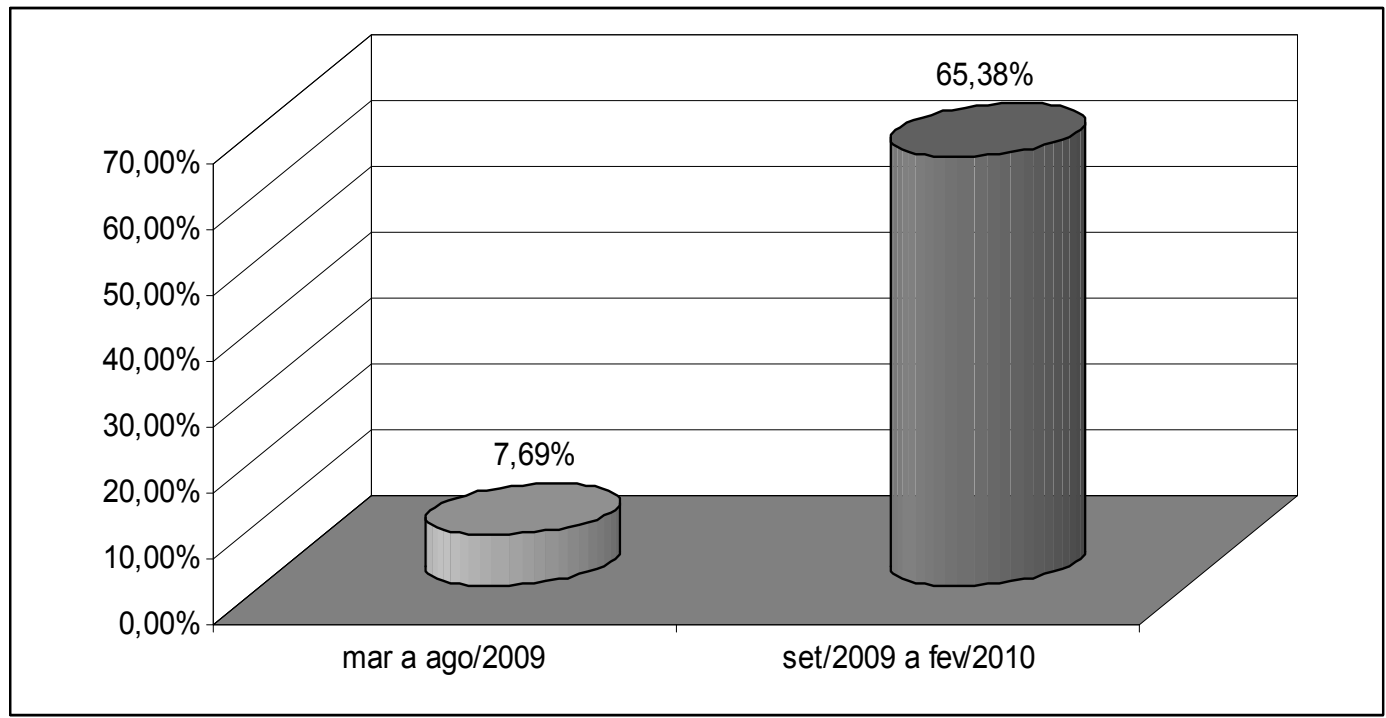

Figura 3 - Distribuição porcentual dos RN que realizaram o reteste no período de março/2009 a fevereiro/2010 no município de Sobral-Ce. Sobral-CE, 2010

Percebeu-se que esta incidência de falha $(39,77 \%)$ apresentou-se elevada considerando o estudo comparativo realizado na Associação de Pais e Amigos dos Excepcionais (APAE) de Minas Gerais. Nesta investigação foram avaliados 100 RN com e sem risco auditivo, sendo que apenas $9,0 \%$ falhou no teste. Por meio dessa pesquisa concluíram que não houve diferença significante entre os resultados do teste de $\mathrm{RN}$ com e sem risco auditivo ${ }^{9}$.
Em decorrência da grande procura pelo teste da orelhinha nos CSF, a TAN realizada pelo SASA prioriza os $\mathrm{RN}$ de risco, respeitando o princípio da eqüidade do SUS. No entanto, mesmo dando prioridade aos RN de risco, os enfermeiros da ESF ainda encaminham crianças sem fator de risco para o teste da orelhinha.

Percebeu-se que a TAN quando realizada somente nas crianças que apresentam IRDA acarreta uma falha de identificar $50 \%$ dos deficientes 
auditivos, uma vez que até $50 \%$ dos RN com perda auditiva não têm nenhum fator de risco ${ }^{10}$. Muitas crianças sem IRDA não são diagnosticadas até que completem 18 meses de vida ${ }^{11}$, o que acaba acarretando prejuízo no desenvolvimento da fala e linguagem dessas crianças, e é por esse motivo que é preconizada sua realização de forma universal, ou seja, em mais de $95 \%$ dos $\mathrm{RN}^{5}$.

$\mathrm{Na}$ amostra, observou-se que a maioria das crianças que falharam no teste não apresentou nenhum risco auditivo. Sabe-se que as prováveis causas de falhas nos teste deve-se aos locais com barulho, as descamações celulares presentes no CAE, presença de secreção em orelha média, respiração ruidosa, agitação da criança, rolha de cera e gripes ${ }^{12}$. Essas falhas acabam gerando ansiedade nos pais sobre uma provável deficiência auditiva em seus filhos. Reforça-se aqui, que a orientação deve ser dada aos pais quanto ao processo de desenvolvimento normal da linguagem e o comportamento da audição de uma criança nesse processo, para que comunique ao médico ou enfermeira qualquer sinal de alteração, a fim de diminuir o stress psicológico que a família é submetida.

Ressalta-se ainda, a importância da presença do fonoaudiólogo nos cursos de gestantes para fornecer orientações como: cuidados na hora do banho; sobre o uso de cotonetes, grampos, palitos e outros objetos no ouvido; posição adequada de amamentar; cuidados com as infecções das vias áreas superiores; otites médias de repetição/persistente, com efusão por pelo menos três meses e acompanhamento do comportamento auditivo dos lactentes.

O curso de gestantes acontece durante o acompanhamento pré-natal, período em que todas as orientações prestadas pela equipe, assim como as informações sobre gestante devem ser registradas no prontuário.

A experiência de levantamento de dados nos prontuários no CSF do Sumaré mostrou dificuldades na coleta de informações sobre as condições de nascimento dos bebês. Verificou-se que o preenchimento dos dados não é realizado de forma completa pelas enfermeiras e tal situação contribuiu negativamente para o levantamento dos riscos neonatais.

Conforme os achados, 2 prontuários não continham registros do tipo de parto e em 9 prontuários não havia a idade gestacional dos bebês, apesar da literatura não apresentar nenhuma correlação de dados entre o tipo de parto e alterações auditivas. Encontrou-se apenas relação de alterações auditivas com a prematuridade.

Para caracterização dos $\mathrm{RN}$ foram pesquisados os relatórios mensais de devolutiva do SASA ao
CSF Sumaré contendo informações sobre a realização do teste da orelhinha. Após esta pesquisa, os prontuários da família foram consultados, a fim de identificar qual o tipo de indicador de risco.

Identificou-se que alguns $\mathrm{RN}$ classificados sem IRDA pelo relatório de devolutiva do SASA apresentavam indicador de risco segundo o prontuário do CSF, como por exemplo, RN que receberam medicamentos ototóxicos no período de internação. Tal dado apontou divergências entre as informações do relatório mensal de devolutiva com os prontuários da unidade. Isso pode ter ocorrido devido ao fato que os RN estavam sendo encaminhados pelos profissionais da ESF sem as devidas informações sobre as condições de nascimento da criança e/ou os profissionais do SASA não estavam colhendo as informações necessárias sobre os riscos neonatais.

Observou-se também, após consultas nos prontuários, que a equipe de saúde considera prematuridade e peso abaixo de $2.500 \mathrm{~g}$ como risco para deficiência auditiva. As crianças com esses fatores são encaminhadas como rotina para o teste da orelhinha pelo serviço em estudo. O Joint Committee on Infant Hearing não faz referência à prematuridade e ao peso abaixo de $2.500 \mathrm{~g}$ como fator de risco para deficiência auditiva ${ }^{5}$. Já em relação a prematuridade e ao peso inferior a $1.500 \mathrm{~g}$ como fator de risco, a literatura apresenta informações contraditórios em relação a incidência de deficiência auditiva ${ }^{13,14}$.

Acredita-se que as alterações auditivas que podem ocorrer em RN pré-termo são devido às complicações médicas que estes apresentam ao nascer e não em relação ao baixo peso especificamente. Diante do exposto, também não foram encontradas literaturas que comprovem a correlação baixo peso de $2.500 \mathrm{~g}$ com alterações auditivas.

Sugere-se implantar no Questionário de Avaliação de Indicadores para Deficiência Auditiva do SASA dados como nome da criança, sexo, idade gestacional e peso ao nascer para que contemple todas as crianças com e sem IRDA e que sejam preenchidos em três vias, no momento da visita domiciliar, uma para ser encaminhada ao SASA junto ao consolidado, que já é rotina, outra para a mãe, a fim de levar no dia do exame e outra para constar no prontuário da criança. Apesar da literatura apresentar informações contraditórias sobre a prematuridade e deficiência auditiva este indicador também deve ser incluído no Questionário, a fim de que estudos mais amplos relacionando baixo peso e alterações auditivas possam ser pesquisados.

Identificou-se que após o início da atuação da fonoaudiologia em setembro/2009, cresceu o número de encaminhamentos para o teste da 
orelhinha implicando num aumento de $8,33 \%$. Mesmo com uma redução nos meses subseqüentes esse índice continua maior do que o período anterior à inserção da fonoaudiologia na ESF.

Verificou-se ainda uma diferença em relação ao número de encaminhados e exames realizados. Percebeu-se que mensalmente, a enfermeira do CSF realiza a consolidação dos dados baseado no livro de marcação de consultas, informando a quantidade de crianças que foram encaminhadas e que realizaram o teste da orelhinha no mês ao SASA.

Entende-se que a consolidação realizada desta maneira pode apontar para um dado não fidedigno, pois os dados do consolidado do CSF não correspondem aos dados informados pelo SASA. Essas divergências acontecem devido à procura espontânea pelo serviço dos bebês nascidos na Santa Casa de Misericórdia de Sobral, o que justifica um maior número de crianças que realizaram o teste em relação ao número de encaminhamentos realizados pelos enfermeiros da ESF.

Acredita-se que, a forma como os dados são consolidados pelo CSF em estudo dificulta o acompanhamento das crianças encaminhadas antes que estas se dirijam ao SASA, pois só se tem conhecimento quem são as crianças quando o relatório mensal de devolutiva é encaminhado ao CSF pelo SASA.

Durante o período do estudo foi criado um mapa de acompanhamento para facilitar a identificação das crianças. Este instrumento deverá ser preenchido pelos enfermeiros, a fim de registrarem todas as crianças que serão encaminhadas para o teste da Orelhinha e para o monitoramento dos retornos pelos profissionais e fonoaudiólogo do CSF do Sumaré de forma que os dados consolidados sejam também qualitativos, bem como anexar uma via desse mapa ao consolidado que será enviado ao SASA.

O mapa irá facilitar o acompanhamento e monitoramento dos $\mathrm{RN}$, principalmente no que se refere ao reteste, pois após a atuação da residente de fonoaudiologia no CSF aumentou a quantidade de crianças que realizou o reteste.

O mecanismo de reteste é importante porque minimiza o número de encaminhamentos de crianças falso-positivos para diagnóstico e as crianças que apresentam IRDA devem ser acompanhadas a cada 6 meses por 3 anos para que se detecte possível perda progressiva ou tardia ${ }^{5}$.

Um estudo realizado no Hospital das Clínicas da Faculdade de Medicina da USP, dos 1127 RN atendidos, 1020 (90,5\%) apresentaram triagem auditiva adequada, enquanto 107 (9,5\%) apresentaram alterações em uma das avaliações. Dos 107 casos com triagem inadequada, 84 casos $(78,5 \%)$ foram encaminhados para reteste, sendo que apenas 32 RN $(38,1 \%)$ compareceram ${ }^{11}$. Considera-se que após a inserção da fonoaudiologia na ESF, a incidência de retorno para o teste da orelhinha $(65,38 \%)$ deste estudo foi satisfatória, em relação à literatura apresentada de $(38,1 \%)$.

O mais difícil na TAN é conseguir a taxa de retorno, uma vez que impede o diagnóstico precoce e posterior intervenção ${ }^{15-16}$. Estudos recentes sugerem que a perda do seguimento esteja associada a fatores como ausência de serviços de qualidade, comunicação inadequada entre os diversos prestadores, falta de fonoaudiólogos pediatras, cobertura inadequada do seguro de saúde, várias características demográficas, falta de informação, a perda auditiva não é percebida como risco de vida, falta de consciência da comunidade sobre a importância da triagem para a detecção da perda auditiva, entre outros ${ }^{17-22}$.

A pouca valorização da prevenção primária e secundária, a falta de conscientização e informação da população sobre a necessidade e importância da detecção precoce da surdez infantil e a reduzida participação das equipes de saúde, principalmente dos pediatras na valorização e no encaminhamento para TAN são possivelmente os fatores responsáveis pelo atraso na suspeita e diagnóstico tardio 23. A participação efetiva de profissionais de saúde envolvidos neste processo, como médicos, enfermeiros ${ }^{15}$ e o agente comunitário de saúde (ACS), são essenciais por estes serem atores integrantes da equipe mínima que assiste o bebê no CSF a partir do nascimento, possibilitando uma ação global à saúde deste.

Recomenda-se que seja desenvolvida uma sessão educativa e aconselhamento durante o prénatal sobre a TAN e sua importância para o desenvolvimento de fala e linguagem, a fim de garantir maior adesão dos cuidadores ao reteste ${ }^{15}$. O acompanhamento das crianças após o nascimento deve ser realizado durante as visitas domiciliares e consulta de puericultura, espaço este o qual enfermeiros e médicos observam todo o desenvolvimento da criança até 2 anos de idade podendo encaminhar e solicitar o teste da orelhinha, já que durante a coleta de dados não se encontrou muitos registros desses resultados nos prontuários. Os ACS também têm papel fundamental durante as visitas domiciliares, ao lembrar os pais o dia do exame, agendando os retornos caso necessário, bem como ressaltando a importância de levar o resultado do teste no dia da consulta de puericultura.

A TAN pode garantir a detecção precoce, o diagnóstico e a reabilitação a tempo de minimizar os efeitos da deficiência auditiva sobre o indivíduo ${ }^{23}$. Cabe aos profissionais estarem atentos na 
investigação dos IRDA, observação do comportamento auditivo normal da criança e encaminhamento das crianças suspeitas de problemas auditivos ao serviço especializado.

\section{CONCLUSÃO}

A partir dos elementos analisados neste trabalho, verificou-se um aumento significante do número de encaminhamentos para o teste da orelhinha e exames realizados após a inserção da fonoaudiologia na ESF.

Em relação à incidência, observou-se que o percentual de falha no teste da orelhinha de um $\mathrm{RN}$ sem risco auditivo é maior do que em $\mathrm{RN}$ com risco auditivo. Esse dado pode ser um importante argumento para que a TAN seja realizada de forma universal a fim de assegurar que as crianças sem indicador de risco sejam diagnosticadas precocemente minimizando os prejuízos para seu desenvolvimento.
As características das crianças que falharam no teste mostraram maior ocorrência de falhas em RN do sexo masculino, tipo de parto cesariana e idade gestacional de 38 a 41 semanas. Ainda em relação a caracterização dos $\mathrm{RN}$ o fator de risco encontrado em maior evidência foi o uso de medicação ototóxica no período de internação. Esses dados não se mostram conclusivos por não ter encontrado literatura que faça correlação entre os achados.

A taxa de retorno do presente estudo mostrouse significativamente maior em relação ao período anterior a atuação da residente de fonoaudiologia. Isso pode ajudar a mudar a percepção dos profissionais envolvidos nesse processo sobre a importância da triagem auditiva neonatal, a fim de detectar precocemente alterações auditivas antes do primeiro ano de vida.

Por meio deste estudo, pôde-se concluir que a presença do fonoaudiólogo na APS é fundamental no acompanhamento e monitoramento do diagnóstico precoce das alterações auditivas, a fim de propiciar melhoria na qualidade de vida das crianças no município.

\begin{abstract}
Purpose: to analyze the monitoring of the newborn and the promotion of hearing health after insertion of speech therapy at the Family Health Strategy. Method: this is a retrospective documentary study with quantitative approach using 88 infants who underwent OAE testing in the period from February to May 2010, we examined the monthly reports' devolution of the Health Hearing Service of the Municipality, consolidated monthly statements and the handbooks of the Center for Family Health in Sobral-Ce. Results: from the studied newborns, 35 (39.77\%) failed the test, 7 (20\%) were infants with risk indicator for hearing loss and $28(80 \%)$ did not have any risk; It was found that some infants classified by risk indicator of the health Hearing Service had no risk factor according to the Family Health Center. It was also observed that increased the number of referrals to the OAE test increased $8.33 \%$. As for the retesting, $1(7.69 \%)$ child returned in the months from March to August 2009, compared to the months of September/2009 to February/2010 where $17(65.38 \%)$ children had being retested after the performance of speech therapy in the CSF of Sumaré. Conclusion: the presence of speech therapy in primary care is essential for monitoring and tracking the early diagnosis of hearing disorders.
\end{abstract}

KEYWORDS: Health Promotion; Hearing; Speech, Language and Hearing Sciences

\section{REFERÊNCIAS}

1. Oliveira EM et al. A Promoção da Saúde e a Interface com a Educação: a experiência do Município de Sobral - Ceará. In: Brasil. Ministério da Saúde. Escolas promotoras de saúde: experiências do Brasil / Ministério da Saúde, Organização
Pan-Americana da Saúde. Brasília: Ministério da Saúde, 2006; 277-89.

2. Northen JL, Downs MP. Audição na Infância. 5 ed. São Paulo: Guanabara Koogan; 2005.

3. Comitê Brasileiro sobre Perdas Auditivas na Infância - CBPAI. Recomendação 01/99. Jornal do Conselho Federal de Fonoaudiologia. Brasília: CBPAI. 2000; (5):3 -7. 
4. Borges et al. Triagem Auditiva Universal. Arq. Int. Otorrinolaringol. / Intl. Arch. Otorhinolaryngol. São Paulo, 2006; 10(1):28-34.

5. Joint Committee on Infant Hearing. Year 2000 position statement: Principles and guidelines for early hearing detection and intervention program. Pediatrics. 2000; 106(4):798-817.

6. Instituto Brasileiro de Geografia e Estatística-IBGE [homepage]. Cidades: Estimativa da população 2009. [acessado em 20 mar. 2009]. Disponível em: http://www.ibge.gov.br.

7. Sobral. Secretaria de Assistência a Saúde/ DAB-DATASUS-SIAB. Sistema de Informação de Atenção Básica. Sobral, 2010.

8. Widen JE, Bull W, Folsom RC. Newborn Hearing Screening: What it means for providers of early intervention services. LWW/IYC. 2003; 16(3):249-57.

9. Dias FAM, Rezende MCL. Programa de triagem auditiva neonatal e os fatores de risco para deficiência auditiva. Congresso Brasileiro de Fonoaudiologia; 2009 out. 21-24; Salvador, Brasil: Sbfa; c2009. 2246 p. Disponível em http:// www.sbfa.org.br/portal/anais2009/anais_select. php?op=PT\&cid=2246\&tid=1.

10.Joint Commitee on Infant Hearing. 1994 Position statement. Audiology Today. 1994; 6(6):6-9.

11. Pádua FGM et al. Triagem Auditiva Neonatal: Um Desafio para sua Implantação Arq. Otorrinolaringol. São Paulo, 2005; 9(3):190-4.

12. Wittman-Price RA, Pope KA. Universal newborn hearing screening. Am J Nurs. 2002; 102:71-7.

13. Pereira, PKS et al. Programa de triagem auditiva neonatal: associação entre perda auditiva e fatores de risco. Pró-Fono Revista de Atualização Científica, Barueri (SP), 2007; 19(3):267-78.

14. Ari-Evenroth, D et al. Low prevalence of hearing impairment among very low birthweight infants as detected by universal neonatal hearing screening.
Arch Dis Child Fetal Neonatal Ed. 2006; 91(4): F257-62.

15. Kanji A, Shangase KK, Ballot D. Hearing screening follow-up return rate in avery lowbirth weinght project: A retrospective record reviw. Sajch December 2010; 4(4):95-9.

16. Olusanya BO. Follow-up default in a hospitalbased universal newborn hearing screening programme in a low-income country. Child Care Health Dev 2009; 35:190-8.

17. Shulman $S$ et al. Evaluation of the Universal Newborn Hearing Screening and Intervention Program. Pediatrics. 2010; 126(1):S19-27.

18. Liu CL, Farrell J, MacNeil JR, Stone S, Barfield W. Evaluating loss to follow-up in newbornhearing screening in Massachusetts. Pediatrics. 2008; 121(2):e335-43. Disponível em: www.pediatrics. org/cgi/content/full/121/2/e335.

19. Park AH, Warner J, Sturgill N, Alder SC. A survey of parental views regarding their child'shearing loss: a pilot study. Otolaryngol Head Neck Surg. 2006; 134(5):794-800.

20. National Center for Hearing Assessment and Management State summary statistics: universal newborn hearing screening. Disponível em: www. infanthearing.org/suvey/2004statesurvey/results_ obstacles.html.

21. Olusanya BO, Akinyemi OO. Community-based infant hearing screening in a developing country: parental uptake of follow-up services. BMC Public Health. 2009; 9:66.

22. Hatzopoulos S, Qirjazi B, Martini A. Neonatal hearing screening in Albania: results from an ongoing universal screening program. Int $\mathrm{J}$ Audiol. 2007; 46:176-82.

23. Gatto $\mathrm{Cl}$, Tochetto TM. Deficiência Auditiva Infantil: Implicações e Soluções. Rev. CEFAC. 2007; 9(1):110-5.
http://dx.doi.org/10.1590/S1516-18462011005000114

RECEBIDO EM: 03/12/2010

ACEITO EM: 30/04/2011

Endereço para correspondência:

Raquel Martins Maia

Rua Primeiro de Maio, 2501

Teresina - PI

CEP: $64002-510$

E-mail: fga.raquelmaia@ hotmail.com 